PROCEEDINGS OF THE

AMERICAN MATHEMATICAL SOCIETY

Volume 140, Number 2, February 2012, Pages 589-593

S 0002-9939(2011)10956-0

Article electronically published on June 10, 2011

\title{
DIFFEOMORPHISMS WITH GLOBAL DOMINATED SPLITTINGS CANNOT BE MINIMAL
}

\author{
PENGFEI ZHANG
}

(Communicated by Yingfei Yi)

\begin{abstract}
Let $M$ be a closed manifold and $f$ be a diffeomorphism on $M$. We show that if $f$ has a nontrivial dominated splitting $T M=E \oplus F$, then $f$ cannot be minimal. The proof mainly uses Mañé's argument and Liao's selecting lemma.
\end{abstract}

\section{INTRODUCTION}

In 4 Herman constructed a (family of) $C^{\infty}$ diffeomorphism(s) on a compact manifold that is minimal and has positive topological entropy simultaneously. Therefore, positive entropy is insufficient to guarantee the nonminimality. This draws forth the problem of finding some natural structure of the system that is incompatible with the minimality. In 7 Mañé gave an argument to locate some nonrecurrent point if the map admits some invariant expanding foliation (also see [1]). In particular, this argument shows that a partially hyperbolic diffeomorphism always has some nonrecurrent point and hence cannot be minimal. In this paper we show that a global dominated splitting is sufficient to exclude the minimality of the system.

Let $M$ be a closed Riemannian manifold and $f: M \rightarrow M$ be a $C^{1}$ diffeomorphism on $M$. The map $f$ is said to have a global dominated splitting on $M$ if there exist an invariant splitting $T M=E \oplus F$ and two numbers $\lambda \in(0,1)$ and $C \geq 1$ such that

$$
\left\|\left.D f^{n}\right|_{E(x)}\right\| \cdot\left\|\left.D f^{-n}\right|_{F\left(f^{n} x\right)}\right\|<C \lambda^{n} \text { for all } n \geq 1, x \in M \text {. }
$$

A Riemannian metric on $M$ is said to be adapted to the dominated splitting if we can take $C=1$ in (1.1) with respect to this metric. Adapted metrics always exist; see [3] for details.

Although the restriction of the existence of a dominated splitting is much weaker than that of a (partially) hyperbolic splitting, we show that the restriction is strong enough to exclude the possibility of minimality. Recall that the map $f: M \rightarrow M$ is said to be minimal if for each $x \in M$ the orbit $\mathcal{O}_{f}(x)=\left\{f^{n} x: n \in \mathbb{Z}\right\}$ is a dense subset in $M$. The following is our main result.

Received by the editors November 24, 2010.

2010 Mathematics Subject Classification. Primary 37D30.

Key words and phrases. Dominated splitting, minimal, Liao's sifting lemma, Liao's selecting lemma, periodic shadowing.

(C)2011 American Mathematical Society Reverts to public domain 28 years from publication 
Main Theorem. Let $M$ be a closed Riemannian manifold and $f: M \rightarrow M$ be a diffeomorphism on $M$. If $f$ has a global dominated splitting, then $f$ cannot be minimal.

There are vast results in the case that the dimension of $M$ is 2. Pujals and Sambarino gave several good characteristics in 8, 9] for the topology of invariant sets having some dominated splitting for $C^{2}$ diffeomorphisms on surfaces. On the other hand, Xia proved in [11 that for every compact surface $M$ with nonzero Euler characteristic, any homeomorphism on $M$ admits some periodic points. In 12 X. Zhang considered a diffeomorphism $f$ on a closed surface and an $f$-invariant set $\Lambda$ with a dominated splitting. Under some assumptions of $\Lambda$ he showed that there exists a periodic orbit near $\Lambda$ by Liao's selecting lemma and also showed some results about Crovisier's central models.

Our proof combines Mañé's argument and Liao's sifting lemma (or Liao's selecting lemma). Firstly we can find a nonrecurrent point if we are in the case to apply Mañé's argument. Secondly we can find a hyperbolic periodic point if we are in the case to apply Liao's lemmas. Besides these two cases we show that there always exists a proper $f$-invariant subset of $M$. Hence, $f$ is not minimal and our Main Theorem follows.

\section{PARTIAL HYPERBolicity AND QUASI-HYPERBOLIC STRINGS}

In this section we review several useful results for later discussions. Let $M$ be a closed Riemannian manifold and $f: M \rightarrow M$ be a $C^{1}$ diffeomorphism on $M$. Let $T M=E \oplus F$ be a dominated splitting on $M$. We always assume the Riemannian metric on $M$ is chosen to be adapted. That is, there exists $\lambda \in(0,1)$ such that

$$
\left\|\left.D f\right|_{E(x)}\right\| \cdot\left\|\left.D f^{-1}\right|_{F(f x)}\right\|<\lambda \text {, for all } x \in M \text {. }
$$

In this case we say $T M=E \oplus F$ is a $\lambda$-dominated splitting. Observe that $\left\|\left.D f^{n}\right|_{E(x)}\right\| \leq \prod_{k=0}^{n-1}\left\|\left.D f\right|_{E\left(f^{k} x\right)}\right\|$ for all $n \geq 1$. We have similar observations for the subbundle $F$.

The first result we recall is an argument due to Mañé (see [7, Lemma 5.2]) which can locate a nonrecurrent point. Also see [1, Corollary 1].

Proposition 2.1. Let $f$ be a diffeomorphism on $M$ and $\mathcal{W}$ be an $f$-invariant foliation tangent to a distribution $E \subset T M$ such that $D f$ is uniformly expanding (or uniformly contracting) on E. Then there exists a nonrecurrent point of $f$. Moreover, the set $\{z \in M: z \notin \omega(z)\}$ of points that are nonrecurrent in the future is dense in every leaf of $\mathcal{W}$.

Let's sketch the proof here. Without loss of generality we assume $E$ is $\nu$ expanding for some $\nu>1$. First we show that each leaf $W(x)$ contains at most one periodic point. Suppose that there are two periodic points $p, q \in W(x)$ for some $x \in M$. Pick some $n \geq 1$ with $f^{n} p=p$ with $f^{n} q=q$. Let $\gamma$ be a smooth curve in $W(x)$ connecting $p$ and $q$ with length $|\gamma| \leq \nu^{1 / 2} \cdot d_{W}(p, q)$. Then $W(x)$ is $f^{n}$-invariant and $f^{-n} \gamma$ is also a path connecting $p$ and $q$ in $W(x)$ with length $d_{W}(p, q) \leq\left|f^{-n} \gamma\right| \leq \nu^{-n} \cdot|\gamma| \leq \nu^{-1 / 2} \cdot d_{W}(p, q)$. This is impossible unless $p=q$. This shows that each leaf $W(x)$ contains at most one periodic point. Let $N \geq 1 \mathrm{such}$ that $\nu^{N} \geq 5$. Then for a nonperiodic point $y \in W(x)$ we pick $\epsilon>0$ small enough such that $f^{k} B(y, \epsilon), 0 \leq k \leq N$, are pairwise disjoint. Then choose $\delta>0$ to be much smaller than $\epsilon$. We define inductively a sequence of closed disks $D_{n} \subset f^{n} W(y, \delta)$ for 
$n \geq 1$ with $D_{n+1} \subset f D_{n}$ and $D_{n} \cap B(y, 2 \delta)=\emptyset$. Then the intersection $\bigcap_{n \geq 1} f^{-n} D_{n}$ is nonempty, and each point $z \in \bigcap_{n \geq 1} f^{-n} D_{n}$ satisfies $f^{n} z \notin B(y, 2 \delta)$ for all $n \geq 1$. Such $z$ is nonrecurrent, and we finish the proof. See [1, Lemma 5.2] for more details about the construction of $D_{n}$.

The second one is Liao's Sifting Lemma (see [5, 6]), which helps us to locate some periodic point. Also see [10, Lemma 2.2].

Proposition 2.2. Let $1 \leq I \leq d-1$ and $\Lambda$ be a compact $f$-invariant subset of $M$ with a $\lambda$-dominated splitting $T_{\Lambda} M=E \oplus F$ of index $I$. Assume the following:

(1) There is a point $b \in \Lambda$ satisfying $\prod_{k=0}^{n-1}\left\|\left.D f\right|_{E\left(f^{k} b\right)}\right\| \geq 1$ for all $n \geq 1$.

(2) (The tilde condition.) There are $\lambda_{1}$ and $\lambda_{2}$ with $\lambda<\lambda_{1}<\lambda_{2}<1$ such that if a point $x \in \Lambda$ satisfies $\prod_{k=0}^{n-1}\left\|\left.D f\right|_{E\left(f^{k} x\right)}\right\| \geq \lambda_{2}^{n}$ for all $n \geq 1$, then the omega set $\omega(x)$ contains a point $c$ satisfying $\prod_{k=0}^{n-1}\left\|\left.D f\right|_{E\left(f^{k} c\right)}\right\| \leq \lambda_{1}^{n}$ for all $n \geq 1$.

Then for each $\lambda_{3} \in\left(\lambda_{2}, 1\right)$ and each $l \in \mathbb{N}$, there are $l$ positive integers $n_{1}<$ $n_{2}<\cdots<n_{l}$ with the following property: for every $j=1, \cdots, l-1$ and every $k=n_{j}+1, \cdots, n_{j+1}$,

$$
\prod_{i=n_{j}}^{k-1}\left\|\left.D f\right|_{E\left(f^{i} b\right)}\right\| \leq \lambda_{3}^{k-n_{j}} \text { and } \prod_{i=k-1}^{n_{j+1}-1}\left\|\left.D f\right|_{E\left(f^{i} b\right)}\right\| \geq \lambda_{2}^{n_{j+1}-k+1} .
$$

Note that the point $b$ in the first condition of Proposition 2.2 satisfies the assumption of the tilde condition. So the tilde condition is a nontrivial restriction of the proposition.

In the following we sketch how to find a hyperbolic periodic point near $\Lambda$. Proposition 2.2 shows that there are many 'double' uniform stings when $f^{n} b$ approaches some 'good' point $c \in \omega(b)$ : for each $\lambda_{3} \in\left(\lambda_{2}, 1\right)$ and each $l \in \mathbb{N}$, there are $l$ positive integers $n_{1}<n_{2}<\cdots<n_{l}$ with the following property: for every $j=1, \cdots, l-1$ and every $k=n_{j}+1, \cdots, n_{j+1}$,

$$
\prod_{i=n_{j}}^{k-1}\left\|\left.D f\right|_{E\left(f^{i} b\right)}\right\| \leq \lambda_{3}^{k-n_{j}} \text { and } \prod_{i=k-1}^{n_{j+1}-1}\left\|\left.D f\right|_{E\left(f^{i} b\right)}\right\| \geq \lambda_{2}^{n_{j+1}-k+1} .
$$

Then by $\lambda$-domination assumption we have that

$$
\prod_{i=k-1}^{n_{j+1}-1}\left\|\left.D f^{-1}\right|_{F\left(f^{i+1} b\right)}\right\| \leq \prod_{i=k-1}^{n_{j+1}-1} \frac{\lambda}{\left\|\left.D f\right|_{E\left(f^{i} b\right)}\right\|} \leq\left(\frac{\lambda}{\lambda_{2}}\right)^{n_{j+1}-k+1}
$$

for every $k=n_{j}+1, \cdots, n_{j+1}$ and every $j=1, \cdots, l-1$. Let $\tilde{\lambda}=\max \left\{\sqrt{\lambda}, \lambda_{3}, \lambda / \lambda_{2}\right\}$ $<1$. Then the segment $\left(f^{n_{j}} b, f^{n_{j+1}} b\right)=\left\{f^{k} b: n_{j} \leq k \leq n_{j+1}\right\}$ forms a ' $\tilde{\lambda}$-quasihyperbolic string' (see [2]) for each $j=1, \cdots, l-1$.

Let $L \geq 1$ and $d_{0}$ be given by [2, Theorem 1.1] with respect to $\tilde{\lambda}$. For $\epsilon \epsilon$ $\left(0, d_{0}\right]$ let's pick an integer $l=l(\epsilon) \geq 1$ large enough such that given arbitrary $l$ points $x_{1}, \cdots, x_{l}$ in $M$, there exist $i$ and $j$ with $1 \leq i<j \leq l$ such that $d\left(x_{i}, x_{j}\right)<\epsilon$. For this $l$ we let $n_{1}<\cdots<n_{l}$ be given by Proposition 2.2 such that (2.1) holds. Then $d\left(f^{n_{i}} b, f^{n_{j}} b\right)<\epsilon$ for some $1 \leq i<j \leq l$. Therefore, $\left\{\left(f^{n_{i}} b, f^{n_{i+1}} b\right),\left(f^{n_{i+1}} b, f^{n_{i+2}} b\right), \cdots,\left(f^{n_{j-1}} b, f^{n_{j}} b\right)\right\}$ forms a periodic $\tilde{\lambda}$-quasihyperbolic $\epsilon$-pseudo-orbit', and we can apply Liao-Gan's shadowing lemma (see [2, Theorem 1.1]) to find a hyperbolic periodic point $x$ whose orbit $L \epsilon$-shadows the 
periodic pseudo-orbit $\left\{\left(f^{n_{i}} b, f^{n_{i+1}} b\right),\left(f^{n_{i+1}} b, f^{n_{i+2}} b\right), \cdots,\left(f^{n_{j-1}} b, f^{n_{j}} b\right)\right\}$. Clearly this periodic orbit lies in the $L \epsilon$-neighborhood of $\Lambda$. For more details see [2, 10. Also see Liao's Selecting Lemma (for example [10, Lemma 2.3]) for more information.

\section{Dominated SPlitTing AND Minimality}

With the preparations in Section 2 we prove the main theorem that if the map $f$ has a global dominated splitting, then it cannot be minimal.

Proof of the Main Theorem. Let $T M=E \oplus F$ be a $\lambda$-dominated splitting for some $\lambda \in(0,1)$ with respect to some adapted Riemannian metric. If $F$ is uniformly expanding, then $F$ is uniquely integrable and tangent to the strongly unstable foliation $\mathcal{W}^{s u}$. By Proposition 2.1 there exists some nonrecurrent point of $f$, and hence $f$ cannot be minimal on $M$. Similarly, if $E$ is uniformly contracting, then $f$ cannot be minimal either. Then we are left with the case that neither $E$ is uniformly contracting nor $F$ is uniformly expanding. In this case we have that:

(1) since $E$ is not uniformly contracting, there exists $p \in M$ such that $\left\|\left.D f^{n}\right|_{E(p)}\right\| \geq 1$ for all $n \geq 1$

(2) since $F$ is not uniformly expanding, there exists $q \in M$ such that $\left\|\left.D f^{-n}\right|_{F\left(f^{n} q\right)}\right\| \geq 1$ for all $n \geq 1$.

We first observe that, by the $\lambda$-domination assumption, for all $n \geq 1$,

$$
\prod_{k=0}^{n-1}\left\|\left.D f\right|_{E\left(f^{k} q\right)}\right\| \leq \prod_{k=0}^{n-1} \frac{\lambda}{\left\|\left.D f^{-1}\right|_{F\left(f^{k+1} q\right)}\right\|} \leq \frac{\lambda^{n}}{\left\|\left.D f^{-n}\right|_{F\left(f^{n} q\right)}\right\|} \leq \lambda^{n} .
$$

Also note that the first condition in Proposition 2.2 is already satisfied if we take $\Lambda=M$ and $b=p$ since $\prod_{k=0}^{n-1}\left\|\left.D f\right|_{E\left(f^{k} p\right)}\right\| \geq\left\|\left.D f^{n}\right|_{E(p)}\right\| \geq 1$ for each $n \geq 1$. Then we divide the discussion into two subcases:

Subcase I. The tilde condition in Proposition 2.2 holds on $M$ for some $\lambda_{1}, \lambda_{2}$ with $\lambda<\lambda_{1}<\lambda_{2}<1$. Then by Proposition 2.2 and Liao-Gan's shadowing lemma (Theorem 1.1 in 2]), there does exist a hyperbolic periodic point of $f$ : the map $f$ cannot be minimal.

Subcase II. The tilde condition fails. Hence, for each pair $\lambda_{1}, \lambda_{2}$ with $\lambda<\lambda_{1}<$ $\lambda_{2}<1$, there exists some point $\tilde{x} \in M$ such that

- $\prod_{k=0}^{n-1}\left\|\left.D f\right|_{E\left(f^{k} \tilde{x}\right)}\right\| \geq \lambda_{2}^{n}$ for all $n \geq 1$,

- for each $y \in \omega(\tilde{x})$, there exists some $n(y) \geq 1$ with $\prod_{k=0}^{n(y)-1}\left\|\left.D f\right|_{E\left(f^{k} y\right)}\right\| \geq$ $\lambda_{1}^{n(y)}$.

According to (3.1), we see that $q \notin \omega(\tilde{x})$ since $\lambda<\lambda_{1}$. Therefore, $\omega(\tilde{x}) \subsetneq M$ for some point $\tilde{x} \in M$, and the map $f$ is not minimal either.

This finishes the verification for both subcases and ends the proof of the theorem.

Remark 3.1. The result is not true if we consider invariant subsets instead of the whole manifold, since there are various kinds of minimal subsets on which the map $f$ has a dominated splitting with respect to the ambient system. For example, let 
$A=\left(\begin{array}{ll}2 & 1 \\ 1 & 1\end{array}\right)$ and $f_{A}: \mathbb{T}^{2} \rightarrow \mathbb{T}^{2}$ be the induced diffeomorphism. Let $R: \mathbb{T} \rightarrow \mathbb{T}$ be an irrational rotation. Then $f_{A}$ is Anosov with a fixed point $o \in \mathbb{T}^{2}$ and $R$ is minimal. Moreover, the product system $\left(R, f_{A}\right): \mathbb{T} \times \mathbb{T}^{2} \rightarrow \mathbb{T} \times \mathbb{T}^{2}$ is partially hyperbolic with an invariant minimal subset $\Lambda=\mathbb{T} \times\{o\}$.

\section{ACKNOWLEDGMENTS}

This work was done while the author was visiting Peking University. He would like to thank Shaobo Gan, Lan Wen and Zhihong Xia for discussions and suggestions. The author especially thanks Baolin He and Peng Sun for many helpful explanations.

\section{REFERENCES}

1. K. Burns, F. Rodriguez Hertz, M. Rodriguez Hertz, A. Talitskaya and R. Ures, Density of accessibility for partially hyperbolic diffeomorphisms with one-dimensional center, Discrete Contin. Dyn. Syst., 22 (2008), 75-88. MR2410948(2010a:37054)

2. S. Gan, A generalized shadowing lemma, Discrete Contin. Dyn. Syst., 8 (2002), 627-632. MR 1897871 (2003d:37028)

3. N. Gourmelon, Adapted metrics for dominated splittings, Ergod. Th. Dynam. Sys., 27 (2007), 1839-1849. MR2371598(2008k:37070)

4. M. Herman, Construction d'un difféomorphisme minimal d'entropie topologique non nulle, Ergod. Th. Dynam. Sys., 1 (1981), 65-76. MR627787 (83c:58046)

5. Shantao Liao, On the stability conjecture, Chinese Annals of Math., 1 (1980), 9-30. MR591229 (82c:58031)

6. Shantao Liao, An existence theorem for periodic orbits, Acta Sci. Natur. Univ. Pekinensis, (1979), 1-20. MR560169 (82b:58074)

7. R. Mañé, Contributions to the stability conjecture, Topology, 4 (1978), 383-396. MR.516217 (84b:58061)

8. E. Pujals and M. Sambarino, Homoclinic tangencies and hyperbolicity for surface diffeomorphisms, Ann. Math., 151 (2000), 961-1023. MR1779562 (2001m:37057)

9. E. Pujals and M. Sambarino, On the dynamics of dominated splitting, Ann. Math., 169 (2009), 675-740. MR2480616 (2010b:37087)

10. L. Wen, The selecting lemma of Liao, Discrete Contin. Dyn. Syst., 20 (2008), 159-175. MR2350064 (2008i:37058)

11. Z. Xia, Area-perserving surface diffeomorphisms, Commun. Math. Phys., 263 (2006), 723-735. MR.2211821 (2009g:37039)

12. X. Zhang, "Some comments on the proof of 2-dimensional Palis density conjecture" (in Chinese), Master thesis, Peking University, 2010.

Department of Mathematics, University of Science and Technology of China, Hefei, Anhui 230026, People's Republic of China

Current address: CEMA, Central University of Finance and Economics, Beijing 100081, People's Republic of China

E-mail address: pfzh311@gmail.com 